\title{
Assessing clinical benefit response in the treatment of gastric malignant ascites with non-measurable lesions: a multicenter phase II trial of paclitaxel for malignant ascites secondary to advanced/recurrent gastric cancer
}

\author{
Haruhiko Imamoto $\cdot$ Koji Oba $\cdot$ Junichi Sakamoto $\cdot$ Hiroyasu Iishi $\cdot$ \\ Hiroyuki Narahara - Takeyoshi Yumiba - Takashi Morimoto • Masaki Nakamura • \\ Noboru Oriuchi · Chieko Kakutani · Satoshi Morita · Hitoshi Shiozaki
}

Received: 30 September 2010/Accepted: 21 November 2010/Published online: 17 February 2011

(c) The International Gastric Cancer Association and The Japanese Gastric Cancer Association 2011

\begin{abstract}
Background Paclitaxel has shown promise against advanced gastric cancer and associated malignant ascites with non-measurable lesions. In order to evaluate the therapeutic effect of paclitaxel against malignant gastric ascites, a prospective phase II clinical trial was designed according to our previously proposed criteria represented by the clinical benefit response in gastric cancer (CBR-GC) criteria and the five-point method (5PM).

Methods Patients with advanced gastric cancer with malignant ascites were treated with 1-h intravenous (i.v.) infusions of $80 \mathrm{mg} / \mathrm{m}^{2}$ of paclitaxel weekly over a 3-week cycle on days 1,8 , and 15 , followed by 1 week of rest.
\end{abstract}

H. Imamoto $\cdot$ H. Shiozaki

Department of Surgery, Kinki University, Osaka, Japan

K. Oba

Translational Research and Clinical Trial Center Hokkaido

University Hospital, Hokkaido University, Sapporo, Japan

J. Sakamoto $(\bowtie)$

Young Leaders' Program in Health-Care Administration, Department of Social-Life Science, Nagoya University Graduate

School of Medicine, 65 Tsurumaicho, Showaku,

Nagoya 466-8550, Japan

e-mail: sakamjun@med.nagoya-u.ac.jp

H. Iishi

Department of Internal Medicine, Osaka Medical Center

for Cancer and Cardiovascular Diseases, Osaka, Japan

H. Narahara

Department of Clinical Oncology, Hiroshima University,

Hiroshima, Japan

T. Yumiba

Department of Surgery, Osaka Kousei Nenkin Hospital,

Osaka, Japan
Therapeutic responses were measured according to the CBR-GC criteria and the 5PM.

Results The CBR-GC criteria showed improved ascites volume and functional status in $39.1 \%$ of patients. A positive CBR-GC response in abdominal girth was seen in $31.3 \%$ of patients, and this was significantly correlated with the 5PM-estimated change in ascites volume $(p<0.001)$. The median number of treatment cycles was 3 (range 1-12). The most common non-hematological toxicity was anorexia, in $22.2 \%$ of patients.

Conclusion Weekly i.v. paclitaxel is a safe and effective chemotherapeutic regimen based on validated CBR-CG criteria.

\section{T. Morimoto}

Department of Surgery, Yao City Hospital, Yao, Japan

M. Nakamura

Department of Surgery, Wakayama Medical University,

Wakayama, Japan

N. Oriuchi

Department of Diagnostic Radiology and Nuclear Medicine,

Gunma University, Maebashi, Japan

C. Kakutani

EBM Research Center, Kyoto University Graduate School of Medicine, Kyoto, Japan

S. Morita

Department of Biostatistics, Yokohama City University, Yokohama, Japan 
Keywords Gastric cancer - Malignant ascites .

Paclitaxel - Clinical benefit response in gastric cancer $($ CBR-GC) $\cdot$ Five point method (5PM)

\section{Introduction}

Although the incidence of gastric cancer is declining in many industrialized countries, it remains the second-most frequent cause of overall cancer mortality worldwide, and is particularly prevalent in East Asia, as the leading or second-leading cause of cancer mortality in Japan, Korea, and China [1]. Even when patients receive best supportive care, the prognosis for advanced or recurrent gastric cancer is very poor, with a median survival time (MST) of 3-4 months [2-4]. In particular, malignant ascites in terminal gastric cancer is a severe endstage manifestation of the disease and poses particular problems to clinicians in providing suitable palliative care.

Despite the worldwide prevalence of gastric cancer and difficulty with the management of malignant ascites in the terminal stages of the disease, the number of reports dealing with the diagnosis and treatment of gastric malignant ascites is limited. With regard to the treatment of peritoneal dissemination and/or ascites caused by gastric cancer metastasis, there has been no established treatment, partly resulting from the frequent lack of measurable lesions in gastric malignant ascites, preventing assessment by the standard response evaluation criteria in solid tumors (RECIST). This lack of an established chemotherapeutic regimen and the limited efficacy of older anticancer agents against malignant ascites secondary to gastric cancer highlights the need for newer, more effective chemotherapeutic agents and approaches.

Paclitaxel is one of a number of newer agents that have shown promise against advanced gastric cancer and associated malignant ascites, with promising response rates [5-7], a reduced side-effect profile [5-7], and favorable pharmacokinetics [8-10]. Here, we report the first prospective phase II trial of paclitaxel in the treatment of malignant ascites secondary to advanced or recurrent gastric cancer with non-measurable lesions. To evaluate the response of malignant ascites to anticancer therapy objectively, we used two separate assessment methods; the clinical benefit response in gastric cancer (CBR-GC) and the five-point method (5PM). The concept of "clinical benefit response" is broadly accepted and is employed in pancreatic cancer [11-13]. For this study, we prospectively defined the CBR-GC [14] as a composite endpoint of the two parameters of a change in ascites volume (measured by abdominal girth) and functional impairment, in patients with gastric malignant ascites. On the other hand, the 5PM has been validated [15] and used [16] as a simple, accurate, and objective measure of changes in ascites volume in advanced gastric cancer. The present "gold standard" for quantitating ascites volume is surface rendering by threedimensional computed tomography (3D-CT), a method commonly used to estimate the volume of organs and ascites before and after liver transplantation [10, 11, 17, 18]. A practical limitation of the 3D-CT method is that it requires specialist equipment and an appropriately trained radiologist, and more than $30 \mathrm{~min}$ is needed to render images and provide accurate results. In contrast, the 5PM simply requires the measurement of fluid depth at five points on conventional CT images. The estimated ascites volume derived from this method correlates well with results determined by 3D-CT $[9,15]$, indicating that the 5PM may be considered a simple, accurate, and objective method for measuring the volume of ascitic fluid.

The primary objective of this study was to assess the efficacy and safety of weekly intravenous (i.v.) paclitaxel for the treatment of malignant ascites secondary to advanced or recurrent gastric cancer, with the CBR-GC as the primary endpoint; and overall survival, time to treatment failure (TTF), and safety parameters as secondary endpoints. The secondary objective was to prospectively evaluate the correlation between the measurement of abdominal girth as a measure of ascites volume and the ascites volume calculated by the 5PM when using the CBR-GC as a measure of response to treatment in malignant ascites secondary to gastric cancer with non-measurable lesions.

\section{Patients and methods}

\section{Patients}

The design of the study was described in a protocol paper published before the start of this trial [14]. Briefly, patients histologically diagnosed with gastric adenocarcinoma who exhibited clinically relevant ascites for which symptomatic diuretic therapy was ineffective were enrolled. Ascites was clinically diagnosed either by physical examination or by imaging modalities such as abdominal ultrasound (US), $\mathrm{CT}$, or magnetic resonance imaging (MRI). The malignant nature of this ascites was determined by either or both a previous positive pathological diagnosis of gastric cancer or by cytological confirmation from ascitic fluid. Patients with a history of allergy or hypersensitivity reactions to paclitaxel, or synchronous or metachronous malignancy, except for early-stage cervical cancer or early-stage nonmelanoma skin cancer were excluded. Both pretreated and chemotherapy-naive patients were considered to be eligible for the study. In addition, patients with severe complications, such as severe cardiac, liver, or kidney dysfunction 
were considered ineligible, as were patients with a psychiatric disorder or a European Cooperative Oncology Group (ECOG) performance status (PS) of 3 or more.

The study was designed and overseen by a steering committee. The protocol was approved by the protocol review committee of the Japan South West Oncology Group (JaSWOG) and the ethics review committee at each participating institution, according to the principles of the Helsinki Declaration. All patients provided written informed consent. The study was coordinated, and also supported by, the non-profit organization Epidemiological and Clinical Research Information Network (ECRIN).

\section{Treatment}

All patients received a 1-h i.v. infusion of paclitaxel at a dose of $80 \mathrm{mg} / \mathrm{m}^{2}$ weekly over a 3 -week cycle on days 1,8 , and 15 , followed by 1 week of rest. Treatment continued unless there was a dose-limiting toxicity as defined by the National Cancer Institute-Common Toxicity Criteria (NCICTC) version 2.0, objective evidence of tumor progression, an increase in ascites, or it was no longer in the patient's best interest as determined by the treating physicians or the individual patient. Patients completing one cycle of therapy without toxicity received the same dose in the subsequent cycle. At each administration, patients were required to fulfill the following criteria: leukocyte count $2,000 / \mathrm{mm}^{3}$; platelet count $75,000 / \mathrm{mm}^{3}$; hemoglobin $8.0 \mathrm{~g} / \mathrm{dl}$; aspartate aminotransferase (AST) or alanine aminotransferase (ALT) $<2$ times the upper limit of normal; absence of fever; PS 0-2; and absence of non-hematological toxicity of grade 3 or 4 . If any dose-limiting hematological (grade 4 leucopenia, neutropenia, or anemia and greater than grade 3 febrile neutropenia or thrombocytopenia) or non-hematological (grade 3 or greater, except for anorexia, nausea, vomiting, or alopecia) toxicities were present prior to administration, either the subsequent dose of weekly paclitaxel was reduced by $10 \mathrm{mg} / \mathrm{m}^{2}$, or therapy was discontinued entirely, at the discretion of the treating physician.

Evaluation of CBR-GC as the primary endpoint

Each patient was classified as 'positive', 'stable', or 'negative' for each of a change in ascites volume measured and functional impairment, using measures assessed relative to that at registration. The primary measure for determining the ascites volume parameter was abdominal girth, measured from directly superior to the umbilicus, while two secondary measures-abdominal paracentesis frequency, and changes (dosage, timing, type) in diuretic medicationwere assessed when abdominal girth was found to have decreased, to ensure that these clinical factors were not influencing the observed improvement. Specifically,

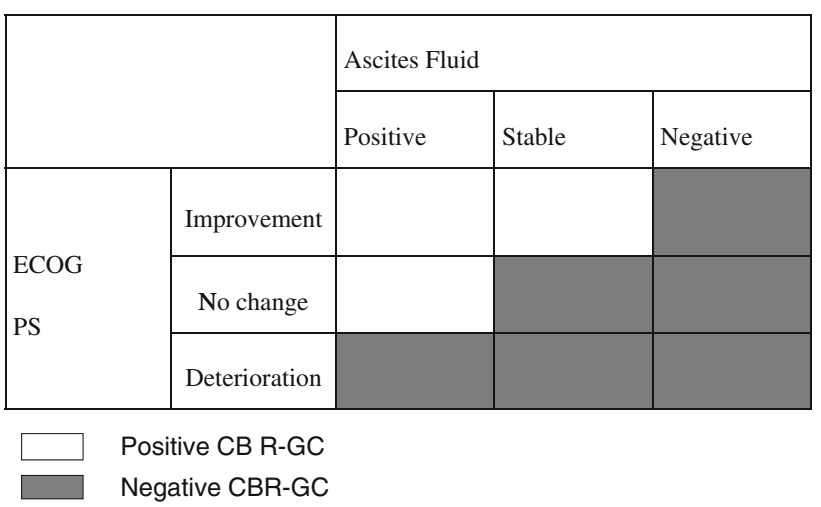

Fig. 1 The definition of clinical benefit response, gastric cancer $(C B R-G C)$. White cell part signifies CBR-GC-positive patients, and the shaded cell part signifies CBR-GC-negative ones. ECOG European Cooperative Oncology Group, PS performance status

a negative designation indicated either or both an increase in abdominal girth and deterioration in either of the remaining two secondary measures; a stable designation indicated no change in abdominal girth and no deterioration in either of the secondary parameters; while a positive designation for ascites volume indicated a decrease in abdominal girth and no deterioration in either of the two secondary parameters. The functional impairment parameter was determined in a similar fashion, but on the basis of change in the ECOG PS, with an improvement designated as positive, no change designated as stable, and deterioration designated as negative, and a change from PS 0 to 1 defined as 'no change'.

To achieve a response in terms of an overall rating of the clinical benefit, patients had to be rated 'positive' for at least one of the two measures (ascites fluid or functional impairment) without being rated 'negative' for another. Otherwise, for the overall rating, patients were classified as being non-responsive (Fig. 1).

Abdominal girth and body weight were measured by either a doctor or nurse every 2 weeks, on days 1 and 15 of each cycle, while CBR-GC status was assessed by both a doctor and nurse independently under the same schedule. Baseline values were taken as those assessed on the first day of the first cycle. If treatment was stopped due to either an increase in ascites or death, the patient was classified as negative for the respective clinical benefit parameter (ascites volume or functional impairment, respectively) and therefore non-responsive to treatment [14]. If treatment was stopped before day 15 of a cycle, values from the last assessment in that cycle were used.

Objective assessment of ascites volume

by the five-point method (5PM)

CT was performed prior to starting the trial and then monthly prior to every treatment cycle to calculate the 


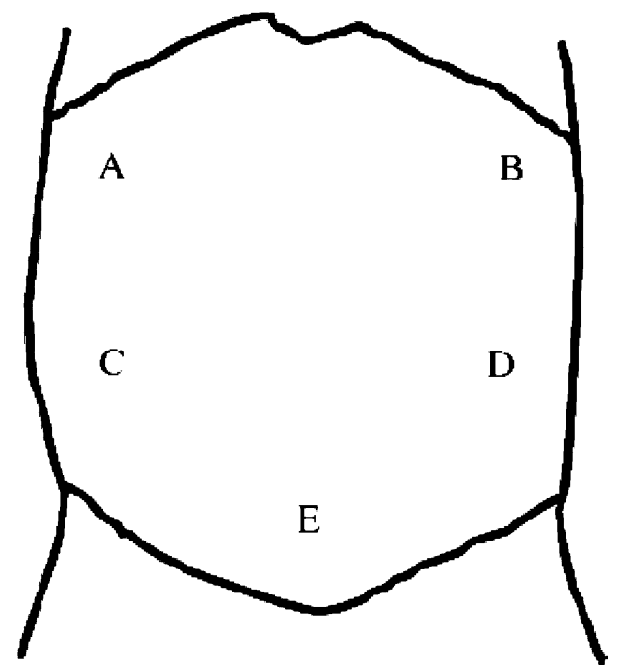

Fig. 2 Measurement of ascites volume using the five-point method (5PM). Ascites volume was accurately estimated using the 5PM as described by Oriuchi et al. [17]. In order to estimate the volume of ascites, the area of a standard abdominal cavity in the anterior projection was assumed to be $1000 \mathrm{~cm}^{2}$. The average thickness of ascites $(A+B+C+D+E) / 5$ was then multiplied by the area to yield the volume of ascites in milliliters. The amount of ascites was calculated by the following equation: $(A+B+C+D+E) \times 200$ $(\mathrm{ml})$

change in ascites volume with the 5PM, as described by Oriuchi et al. [15]. Briefly, fluid depth was measured at five points, corresponding to the left and right subphrenic spaces, paracolic spaces, and the pre-bladder space on images taken at the levels of the superior mesenteric artery root, the lower pole of the left kidney, and the femoral head, respectively. Ascites volume was then calculated as detailed in the legend of Fig. 2 (Oriuchi et al. [15]

\section{Statistical analyses}

The primary endpoint analysis was to estimate the response rate based on the CBR-GC, as explained above. The CBRGC rate was calculated as a proportion with $95 \%$ confidence intervals (CI). In the primary analysis, any positive or negative change in abdominal girth was rated as "positive' or 'negative'. But this rating could be changed if there were possible errors in the measurement of abdominal girth. To account for measurement errors in the abdominal girth, a sensitivity analysis was also undertaken, in which only changes in abdominal girth greater than 5\% were included. In the sensitivity analysis, a negative designation indicated either or both $a \geq 5 \%$ increase in abdominal girth and deterioration in either of the remaining two secondary measures of paracentesis and diuretic therapy; a stable designation indicated an increase or decrease lower than $5 \%$ in abdominal girth and no deterioration in either of the secondary parameters; while a positive designation for ascites volume indicated a $\geq 5 \%$ decrease in abdominal
Table 1 Patient characteristics

\begin{tabular}{ll}
\hline Total no. of patients included in analysis & 64 \\
Sex, $n(\%)$ & $32(50.0)$ \\
Male & $32(50.0)$ \\
Female & \\
Age (years) & 62.0 \\
Mean & $64(32-81)$ \\
Median (range) & \\
ECOG PS, $n(\%)$ & $24(37.5 \%)$ \\
0 & $28(43.8 \%)$ \\
1 & $12(18.8 \%)$ \\
2 & $64(100.0 \%)$ \\
Stage, $n(\%)$ & \\
Disease status, $n(\%)$ & $43(67.1 \%)$ \\
Locally advanced or metastatic & $21(32.8 \%)$ \\
Recurrent & $1(1.6 \%)$ \\
Pretreatment, $n(\%)$ & $18(28.1 \%)$ \\
Preoperative chemotherapy & $18(28.1 \%)$ \\
Postoperative chemotherapy & $27(42.2 \%)$ \\
Other chemotherapy & 2906.1 \\
No chemotherapy & $2796.0(122.4-7623.0)$ \\
Volume of ascites fluid (ml) & \\
Mean & \\
Median (range) &
\end{tabular}

ECOG PS European Cooperative Oncology Group performance status

a Volume of ascites fluid was calculated by the five-point method [2]. Data for two patients are missing from the calculation

girth and no deterioration in either of the two secondary parameters.

Secondary endpoints were overall survival, TTF, change in ascites volume as assessed by the 5PM, and safety parameters. Survival and treatment failure curves were derived by the Kaplan-Meier method, and toxicity was assessed according to the NCI-CTC version 2.0. Correlations between abdominal girth measurement and the 5PM were calculated using Spearman's rank correlation coefficients. All statistical tests were two-sided and were calculated using SAS version 9.1 (SAS Institute, Cary, NC, USA). A $p$ value of $<0.05$ was considered significant.

\section{Results}

Patient characteristics and treatment

Between June 2003 and September 2006, 64 patients (32 males, median age 64 years) with malignant ascites secondary to advanced or recurrent gastric cancer were enrolled, and they were followed for at least 2 years. Forty-three patients $(67.1 \%)$ had histologically confirmed locally advanced or metastatic gastric adenocarcinoma and 
$27(42.2 \%)$ had not received prior chemotherapy (Table 1). The mean volume of ascites assessed by the 5PM was 2,906 $\mathrm{ml}$ at baseline, with considerable inter-patient variation (range 122-7,623 $\mathrm{ml}$ ).

The median number of treatment cycles was 3 (range 1-12), with the most frequent reason for treatment discontinuation being an increase in ascites, seen in 26 patients $(40.6 \%)$. Other reasons for discontinuation were inappropriate treatment continuation as determined by the attending physician in 14 , change of therapeutic strategy in 7 , patient refusal in 5, complications related to tumor progression in 5, hematological toxicity in 3, unknown reasons in 2 , and death in 2 cases.

\section{Treatment response based on CBR-GC}

Abdominal girth was not assessed in five patients due to patient refusal, and these cases were therefore designated as non-responsive to treatment for the primary endpoint analysis. A total of 25 of the 64 patients were deemed to have a positive $\mathrm{CBR}$, based on the $\mathrm{CBR}-\mathrm{GC}$ criteria (CBR$\mathrm{GC}$ rate $=39.1 \%, 95 \%$ confidence interval $(\mathrm{CI})=27.1-$ $51.1 \%$; Table 2). Specifically, 4 patients showed positive improvements in both ascites volume and functional impairment parameters on treatment with weekly paclitaxel, while 21 showed improvement in the ascites volume parameter without an obvious change in functional impairment. The mean change in ascites volume from baseline in these 25 patients was $-2,004 \mathrm{ml}$ (median $-1,912 \mathrm{ml}$ ). In the other 34 patients, the CBR was determined as negative.

Further, in the sensitivity analysis conducted using values with a greater than $5 \%$ change to account for possible errors in abdominal girth measurement, 20 of the 64 patients were deemed to have achieved a positive CBR $(\mathrm{CBR}$ rate $=31.3 \%, 95 \% \mathrm{CI}=19.9-42.7 \%)$. The result of the sensitivity analysis was almost the same as the result of the primary analysis, and it was considered that a $5 \%$ possible error in the measurement of the abdominal girth

Table 2 Clinical benefit response, gastric cancer (CBR-GC)

\begin{tabular}{lllll}
\hline & \multicolumn{2}{l}{ Ascites fluid } & & \\
\cline { 2 - 5 } & Positive & Stable & Negative & Total \\
\hline ECOG PS & 4 & 0 & 2 & \\
Improvement & 21 & 2 & 11 & 6 \\
No change & 8 & 0 & 11 & 34 \\
Deterioration & 33 & 2 & 24 & $59^{\mathrm{a}}$ \\
Total & & & & 19 \\
\hline
\end{tabular}

The CBR-GC rating criteria gave a total of 25 responders and therefore a response rate of $39.1 \%$ (95\% confidence interval $[\mathrm{CI}]=27.1-51.1 \%)$

${ }^{\text {a }}$ Clinical benefit response was not evaluated in five patients did not change the primary results. Details of the threshold percentage change of ascites volume considered optimal for defining "no change" in the ascites volume parameter will be published in a future study.

Correlation of abdominal girth and the five-point method (5PM) as measures of ascites volume

The change in abdominal girth from baseline correlated well with the estimated change in ascites volume as determined by the 5PM (Spearman's rank correlation coefficient $=0.70, p<0.001$; Fig. 3).

Time to treatment failure, overall survival, median survival time, and association with the CBR-GC

As of April 2009, the median TTF was 2.3 months (95\% $\mathrm{CI}=1.8-3.5$; Fig. 4) and 58 of the 64 patients had died, with an MST of 5.2 months (95\% CI = 4.2-9.4; Fig. 5). A clear difference in MST was observed between patients with a positive (9.9 months) versus those with a negative CBR-GC response (3.6 months).

\section{Toxicities}

Hematological and non-hematological toxicities observed in the treatment period are summarized in Table 3.

\section{Discussion}

Here, we describe our use of a multicenter phase II trial of weekly i.v. paclitaxel to prospectively validate novel assessment criteria for determining clinical benefit in the treatment of malignant ascites secondary to gastric cancer with non-measurable lesions. With regard to the primary objective, we found that a positive clinical benefit response (CBR) as determined by the CBR-GC criteria was reflected in an improved MST. Further, the measurement of abdominal girth as a means of assessing changes in ascites volume correlated well with that assessed by the 5PM. With regard to the secondary objectives, our results suggest that weekly i.v. paclitaxel is an effective and safe chemotherapeutic regimen for use in malignant ascites secondary to advanced or recurrent gastric cancer, with a $39.1 \%$ response rate as determined by the CBR-GC criteria, an MST of 5.2 months, and acceptable rates of treatment-related toxicities.

Validity of CBR-GC as a measure of response to treatment in malignant ascites

The assessment of response to treatment in solid malignancies that can either or both manifest at or develop to a 
Fig. 3 Correlation of abdominal girth and five-point method as measures of ascites volume

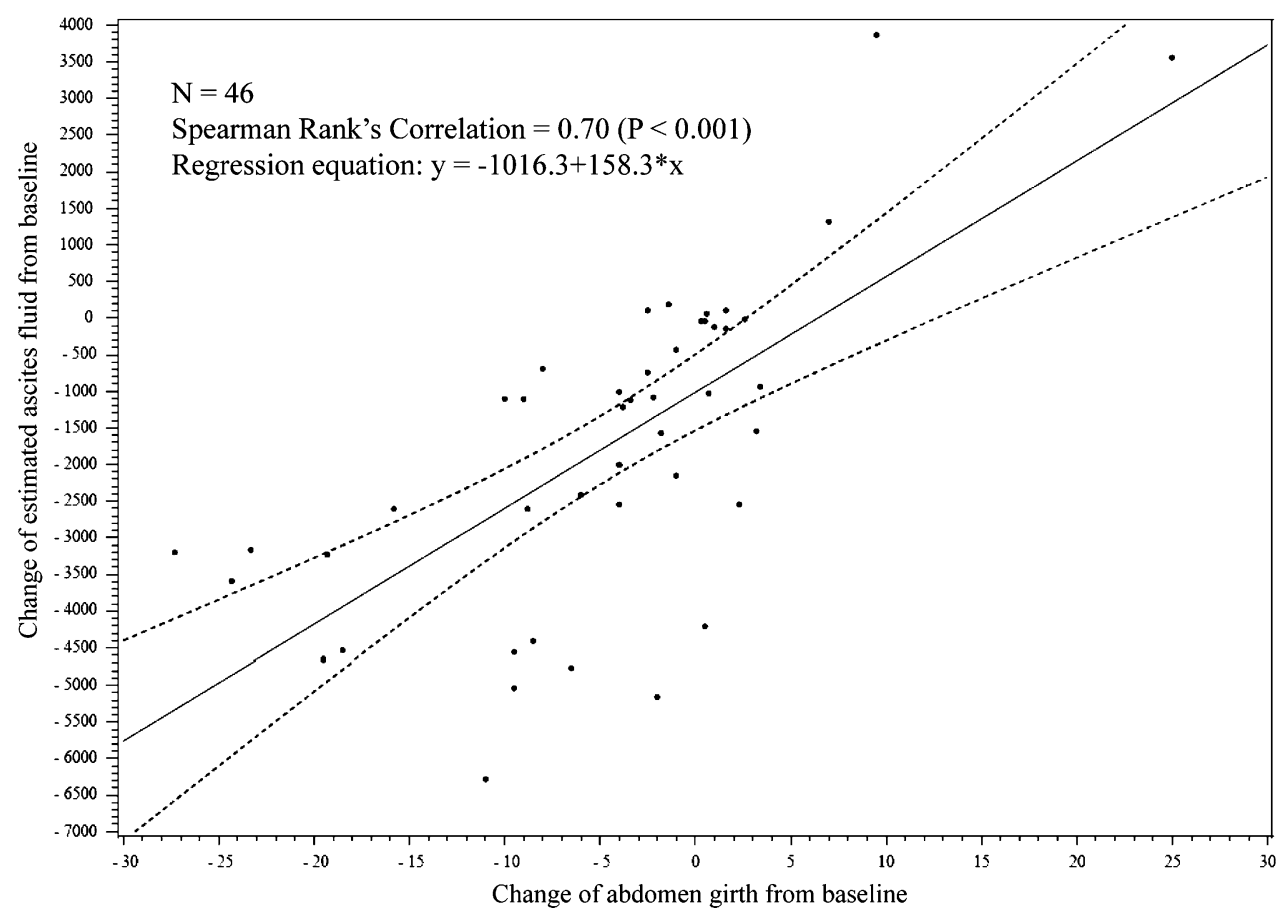

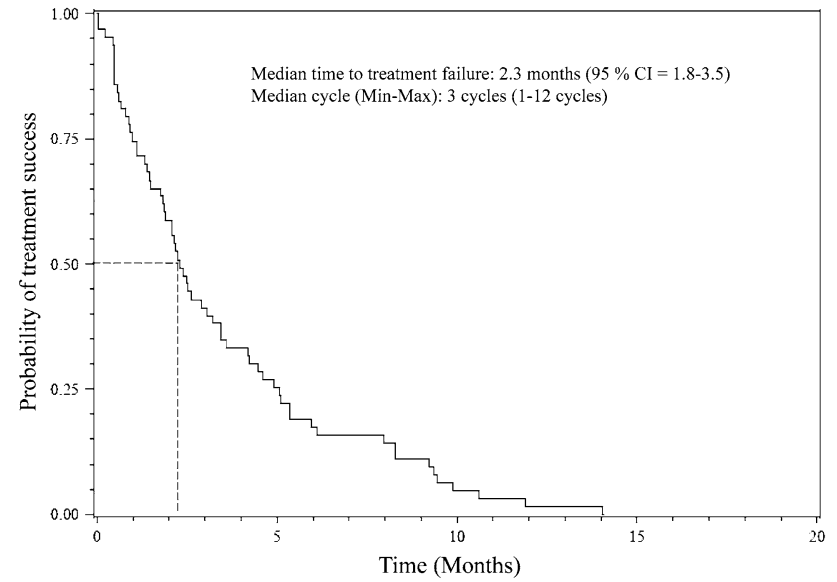

Fig. 4 Kaplan-Meier curve of time-to-treatment failure. CI Confidence interval

terminal stage with non-measurable lesions has been a vexing issue for clinicians and researchers. The current standard for assessing response to treatment by the RECIST requires a measurable lesion of at least $10 \mathrm{~mm}$ diameter if imaged by spiral CT, or at least $20 \mathrm{~mm}$ using conventional techniques. Moreover, the treatment of terminal conditions has a palliative rather than curative intent, and therefore conventional measures of tumor response hold less validity if they do not correlate well with a patient's functional status. The concept of the CBR as a means of assessing response to treatment was first developed in pancreatic cancer, which is often a terminal disease at diagnosis despite the presence of only non-measurable

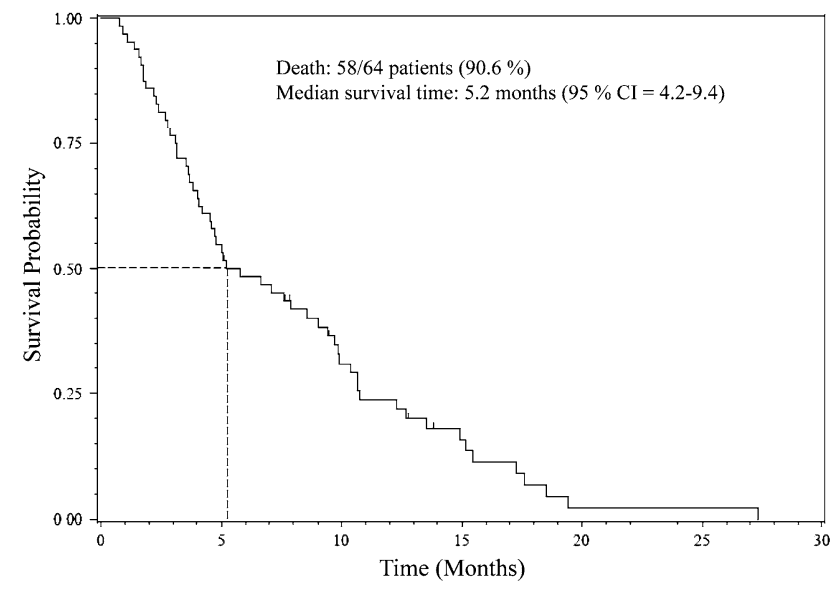

Fig. 5 Kaplan-Meier curve of median survival time

lesions. The original CBR for pancreatic cancer consists of three patient-centered parameters clinically important in the disease; namely, pain, performance status (PS), and weight [11-13]. The CBR is now a widely accepted endpoint in clinical trials for chemotherapeutic agents in pancreatic cancer [18].

Using this CBR criterion for pancreatic cancer as a prototype, we developed the CBR-GC as an objective method of evaluating response to treatment in clinical trials of agents for malignant ascites secondary to gastric cancer with non-measurable lesions. We included two clinically important patient-centered parameters; namely, ascites volume and functional status. Ascites volume was shown to 
Table 3 Toxicities

\begin{tabular}{|c|c|c|c|c|c|c|}
\hline & \multicolumn{6}{|c|}{ NCI-CTC grade } \\
\hline & 0 & 1 & 2 & 3 & 4 & $\geqq 3(\%)$ \\
\hline WBC & 35 & 6 & 16 & 7 & 0 & 10.9 \\
\hline NEU & 40 & 6 & 5 & 10 & 2 & 19.1 \\
\hline LYMPH & 26 & 4 & 20 & 9 & 0 & 15.2 \\
\hline $\mathrm{Hb}$ & 15 & 17 & 21 & 10 & 1 & 17.2 \\
\hline Low $\mathrm{Na}$ & 39 & 11 & 1 & 11 & 1 & 19.1 \\
\hline Nausea & 21 & 16 & 16 & 10 & 0 & 15.9 \\
\hline Vomiting & 44 & 8 & 6 & 5 & 0 & 7.9 \\
\hline Anorexia & 19 & 19 & 11 & 12 & 2 & 22.2 \\
\hline Fatigue & 22 & 22 & 15 & 3 & 1 & 6.5 \\
\hline
\end{tabular}

NCI-CTC National Cancer Institute-common toxicity criteria, NEU neutrophils, $L Y M P H$ lymphocytes, $H b$ hemoglobin

be simply and accurately assessed by changes in abdominal girth measurement. First, we showed that abdominal girth correlated well with ascites volume as assessed by the 5PM. The 5PM has been previously shown to correlate well with results derived from the "gold standard" of measuring ascites volume by 3D-CT rendering [15]. Second, the positive response to treatment as measured by the CBR-GC criteria remained robust after a sensitivity analysis to account for possible errors in measurement of abdominal girth. Further, the CBR-GC took account of potential confounders of this measurement of ascites volume by defining any increase in diuretic consumption or paracentesis frequency as a negative response in this parameter.

Here we showed that the CBR-GC, which employs these two important-patient centered parameters of ascites volume and functional status, was associated with an improved MST in patients with gastric malignant ascites. The CBR-GC may therefore be considered a simple, objective, and accurate measure of CBR in gastric malignant ascites secondary to advanced gastric cancer with nonmeasureable lesions.

Efficacy and safety of paclitaxel for malignant ascites secondary to advanced gastric cancer

Chemotherapeutic treatment of malignant ascites is dependent on the optimal dosage to the peritoneum. This, in turn, highlights the importance of an agent's pharmacokinetics, and particularly its mode of administration. The effectiveness of strategies involving direct intraperitoneal administration of chemotherapeutic or immuno-therapeutic agents, often in combination with hyperthermic therapy, has been widely examined [19-24]. One problem is that most of these agents are rapidly absorbed into the bloodstream on direct administration into the peritoneal cavity, reducing the difference between intraperitoneal and systemic administration and hampering the achievement of higher and more prolonged intraperitoneal concentrations. Distribution may also be hindered by surgical or intraperitoneal injection-related adhesions. These characteristics may explain why a recent large phase III trial of intraperitoneal chemotherapy with older chemotherapeutic agents, including mitomycin $\mathrm{C}$, in Austria and Slovenia was unable to demonstrate a significant effect against advanced gastric cancer [25].

In contrast, recent trials of new anticancer chemotherapeutic agents, administered both individually and in combination, have shown that certain systemic chemotherapy regimens such as oral S-1 (a fluoropyrimidine) and oxaliplatin with low-dose leucovorin and bolus and continuous infusion 5-fluorouracil (modified FOLFOX-4) may also be effective for gastric malignant ascites [16, 25-28]. Paclitaxel is regarded as an active agent, with previous reports indicating that weekly paclitaxel has fewer adverse events and a better safety profile than tri-weekly paclitaxel and may be safely administered to patients with advanced gastric cancer [5-7, 29]. In addition, several studies have presented data on the safety of paclitaxel in recurrent ovarian cancer, with none reporting adverse events specific to patients with ascites [30-32]. Paclitaxel can also be used safely in patients with renal impairment, suggesting that it may be effective in patients whose kidney function is expected to deteriorate in tandem with an increase in ascites as the disease progresses [33]. After i.v. administration, paclitaxel is readily transferred to the ascitic fluid, where its concentration is 1.4 times that in the blood [9], and two pharmacokinetic studies have demonstrated that the concentration remains within the optimal range for $72 \mathrm{~h}$ $[9,10]$. Further, a report on paclitaxel monotherapy also demonstrated complete disappearance of gastric cancerassociated ascites in 3 of 21 patients (14\%) [34]. Recently, in Europe, a bispecific trifunctional antibody, catumaxomab, was approved for the intraperitoneal treatment of malignant ascites in patients with epithelial cell adhesion molecule (EpCAM)-positive tumors [35]. In the treatment of malignant ascites due to gastric cancer, significantly increased puncture-free survival was also reported with catumaxomab [36]. A combination of weekly paclitaxel and catumaxomab in EpCAM-positive gastric malignant ascites might be feasible in future studies.

In the present study, we showed a positive response based on the CBR-GC criteria in 39.1\% (25/64) of patients, a median TTF of 2.3 months, and an overall MST of 158 days (approximately 5.2 months). These results are comparable to those in other trials, such as the Japan Clinical Oncology Group (JCOG) 9603 trial of methotrexate (MTX) and 5-fluorouracil (FU) in treatment-naive patients that showed a disappearance or reduction in ascites as measured by CT or US in $35.1 \%$ (13/37) of patients, 
a median duration of ascites response to therapy of 103 days (approximately 3.4 months), and an MST of 155 days (approximately. 5.1 months) [37], and a trial of modified FOLFOX-4 that showed a disappearance or reduction in ascites as measured by a modified 5PM in $35.4 \%$ (17/48) of patients, a median time to progression of 3.5 months, and an MST of 8.4 months [16].

With regard to toxicities, we found weekly i.v. paclitaxel had an acceptable side-effect profile in patients with gastric malignant ascites. Treatment was discontinued in only 3 patients due to hematological toxicities accompanied by worsening PS, with occurrence rates of grade 4 neutropenia, anemia, leucopenia, and grade 3 or greater febrile neutropenia of $0 \%(n=0), 0 \%(n=0), 1.6 \%$ $(n=1)$, and $3.2 \%(n=2)$, respectively (Table 3$)$ In comparison, the JCOG 9603 trial of MTX and 5-FU showed treatment-related toxicity rates of $5.4 \%$ for grade 4 leucopenia, $10.8 \%$ for grade 4 neutropenia, $8.1 \%$ for grade 3 hyperbilirubinemia, and a $5.4 \%$ rate (2/37) of treatmentrelated deaths [37]. Meanwhile, a modified FOLFOX-4 regimen showed treatment-related toxicity rates of $9.4 \%$ for grade 4 neutropenia and $2.6 \%$ for grade 4 febrile neutropenia [16]. Considering the favorable results relative to other regimens and the already compromised health status of patients with terminal-stage gastric cancer, we believe weekly i.v. paclitaxel has an acceptable side-effect profile in the treatment of gastric malignant ascites.

The survival time seen in the present study and the acceptable side-effect profile of paclitaxel therefore makes this regimen attractive for use in endstage cancer patients with a poor quality of life (QOL). The treatment of advanced gastric cancer-associated malignant ascites by weekly i.v. paclitaxel may be considered a reasonable treatment regimen for this notoriously difficult clinical scenario, which has no established therapeutic protocol.

\section{Limitations}

Several limitations of this study warrant consideration. First, this study had multiple inter-related objectives as it sought to assess both a chemotherapeutic regimen and novel criteria for assessing response to such a regimen in gastric malignant ascites. Given the lack of appropriate established criteria for assessing patient-centered responses to therapy in gastric malignant ascites, this was largely unavoidable. Further, we sought to avoid post-hoc analysis by prospectively defining CBR-GC in a pre-published study protocol [14]. Second, we used the 5PM to assess the accuracy of abdominal girth measurements, rather than the "gold standard" of 3D-CT rendering. Also, our original protocol considered follow-up $\mathrm{CT}$ as optional and did not specifically include the 5PM as a means of assessing ascites volume. All patients underwent regular CT, however, and given our previous study showing a high correlation between the 5PM and 3D-CT rendering [15] and the subsequent demonstration of the convenience and accuracy of the 5PM by others [16], we therefore utilized it in this study as a means of measuring ascites volume and assessing the accuracy of abdominal girth measurement. Additional studies are warranted to further validate both abdominal girth measurement and the CBR-GC as a means of assessing changes in ascites volume and patient-centered response to therapy in gastric malignant ascites, respectively. The present study, however, serves as a suitable 'proof of concept' and justifies further research into and use of the CBR-GC criteria. Third, this was a relatively small, single-arm phase II trial of weekly i.v. paclitaxel and further studies would be required to establish this regimen as safe and effective in patients with malignant ascites due to advanced or recurrent gastric cancer. Fourth, although undertaken in accordance with the original study protocol [14], results concerning QOL data assessed by the Functional Assessment of Cancer Therapy-General (FACT-G) questionnaire were not included here for fear of making this report excessively complex. These QOL results will be published separately in the near future.

\section{Summary}

This multicenter phase II trial of weekly paclitaxel in patients with malignant ascites secondary to recurrent or advanced gastric cancer prospectively validated the novel CBR-GC assessment criteria as a simple, objective, and accurate measure of clinical benefit response (CBR) in cases of malignant ascites secondary to gastric cancer that lack measurable lesions. Further, weekly i.v. paclitaxel was shown to confer that MST and TTF were comparable to those in other studies, and had an acceptable side-effect profile, suggesting that weekly paclitaxel is a safe and effective chemotherapeutic regimen in the treatment of malignant ascites in advanced or recurrent gastric cancer.

Further investigations relating to these findings are warranted, including additional studies using and validating both the CBR-GC and 5PM as means of assessing treatment response in patients without measurable lesions, as well as other trials to compare alternative chemotherapeutic agents, regimens, and modes of administration for the treatment of gastric malignant ascites.

Acknowledgments This study was supported, in part, by the nonprofit organization Epidemiological and Clinical Research Information Network (ECRIN), Japan.

\section{References}

1. Mathers C. Loncar D. Projections of global mortality and burden of disease from 2002 to 2030. PLoS Med. 2006; 3:442. World 
Health Organization, Geneva. http://www.who.int/healthinfo/ statistics/bod_projections2030_paper.pdf.

2. Glimelius B, Hoffman K, Haglund U, Nyrén O, Sjödén PO. Initial or delayed chemotherapy with best supportive care in advanced gastric cancer. Ann Oncol. 1994;5:189-90.

3. Murad AM, Santiago FF, Petroianu A, Rocha PR, Rodrigues MA, Rausch M. Modified therapy with 5-fluorouracil, doxorubicin, and methotrexate in advanced gastric cancer. Cancer. 1993;72:37-41.

4. Pyrhönen S, Kuitunen T, Nyandoto P, Kouri M. Randomised comparison of fluorouracil, epidoxorubicin and methotrexate (FEMTX) plus supportive care with supportive care alone in patients with non-resectable gastric cancer. $\mathrm{Br} \mathrm{J}$ Cancer. 1995;71:587-91.

5. Ohtsu A, Boku N, Tamura F, Muro K, Shimada Y, Saigenji K, et al. A phaseII study of a 3-hour infusion of paclitaxel for advanced gastric cancer. Am J Clin Oncol. 1998;21:416.

6. Kodera Y, Ito S, Mochizuki Y, Fujitake S, Koshikawa K, Kanyama Y, Matsui T, Kojima H, Takase T, Ohashi N, Fujiwara M, Sakamoto J, Akimasa N, Chubu Clinical Cancer Group. A phase II study of weekly paclitaxel as second-line chemotherapy for advanced gastric cancer (CCOG0302 study). Anticancer Res. 2007;27:2667-71.

7. Emi Y, Yamamoto M, Takahashi I, Orita H, Kakeji Y, Kohnoe S, Maehara Y. Phase II study of weekly paclitaxel by one-hour infusion for advanced gastric cancer. Surg Today. 2008;38:1013-20.

8. Wiernik PH, Schwartz EL, Strauman JJ, Dutcher JP, Lipton RB, Paietta E. Phase 1 clinical and pharmacokinetic study of Taxol. Cancer Res. 1987;47:2486-93.

9. Kobayashi M, Sakamoto J, Namikawa T, Okamoto K, Okabayashi T, Ichikawa K, Araki K. Pharmacokinetic study of paclitaxel in malignant ascites from advanced gastric cancer patients. World J Gastroenterol. 2006;12:1412-5.

10. Kobayashi M, Oba K, Sakamoto J, Kondo K, Nagata N, Okabayashi T, Namikawa T, Hanazaki K. Pharmacokinetic study of weekly administration dose of paclitaxel in patients with advanced or recurrent gastric cancer in Japan. Gastric Cancer. 2007;10:52-7.

11. Rothenberg ML, Moore MJ, Cripps MC, Andersen JS, Portenoy RK, Burris HA 3rd, Green MR, Tarassoff PG, Brown TD, Casper ES, Storniolo AM, Von Hoff DD. A phase II trial of gemcitabine in patients with 5-FU-refractory pancreas cancer. Ann Oncol. 1996;7(4):347-53.

12. Burris HA 3rd, Moore MJ, Andersen J, Green MR, Rothenberg ML, Modiano MR, Cripps MC, Portenoy RK, Storniolo AM, Tarassoff P, Nelson R, Dorr FA, Stephens CD, Von Hoff DD. Improvements in survival, clinical benefit with gemcitabine as first-line therapy for patients with advanced pancreas cancer: a randomized trial. J Clin Oncol. 1997;15(6):2403-13.

13. Burris H, Storniolo AM. Assessing clinical benefit in the treatment of pancreas cancer: gemcitabine compared to 5-fluorouracil. Eur J Cancer. 1997;33:818.

14. Sakamoto J, Morita S, Yumiba T, Narahara H, Kinoshita K, Nakane Y, Imamoto H, Shiozaki H. Ascitic Gastric Cancer Study Group of the Japan South West Oncology Group. A phase II clinical trial to evaluate the effect of paclitaxel in patients with ascites caused by advanced or recurrent gastric carcinoma: a new concept of clinical benefit response for non-measurable type of gastric cancer. Jpn J Clin Oncol. 2003;33:238-40.

15. Oriuchi N, Nakajima T, Mochiki E, Takeyoshi I, Kanuma T, Endo K, Sakamoto J. A new, accurate and conventional five-point method for quantitative evaluation of ascites using plain computed tomography in cancer patients. Jpn J Clin Oncol. 2005;35:386-90.

16. Oh SY, Kwon HC, Lee S, Lee DM, Yoo HS, Kim SH, Jang JS, Kim MC, Jeong JS, Kim HJ. A Phase II study of oxaliplatin with low-dose leucovorin and bolus and continuous infusion 5-fluorouracil (modified FOLFOX-4) for gastric cancer patients with malignant ascites. Jpn J Clin Oncol. 2007;37:930-5.

17. Piccoli G, Smania S, Toso F, Borghese L, Pozzi Mucelli R, Vergendo M, et al. Multislice CT anatomy of hepatic artery in patients undergoing liver transplantation using 3D reconstructions. Radiol Med. 2004;108:372-9.

18. Ohorodnyk P, Eisenhauer EA, Booth CM. Clinical benefit in oncology trials: is this a patient-centred or tumour-centred endpoint? Eur J Cancer. 2009;45(13):2249-52.

19. Yonemura Y, Fujimura T, Fushida S, Takegawa S, Kamata T, Katayama K, Kosaka T, Yamaguchi A, Miwa K, Miyazaki I. Hyperthermo-chemotherapy combined with cytoreductive surgery for the treatment of gastric cancer with peritoneal dissemination. World J Surg. 1991;15:530-6.

20. Fujimoto S, Shrestha RD, Kokubun M, Ohta M, Takahashi M, Kobayashi K, Kiuchi S, Okui K, Miyoshi T, Arimizu N. Intraperitoneal hyperthermic perfusion combined with surgery effective for gastric cancer patients with peritoneal seeding. Ann Surg. 1988;208:36-41.

21. Glehen O, Schreiber V, Cotte E, Sayag-Beaujard AC, Osinsky D, Freyer G, François Y, Vignal J, Gilly FN. Cytoreductive surgery and intraperitoneal chemohyperthermia for peritoneal carcinomatosis arising from gastric cancer. Arch Surg. 2004; 139(1):20-6.

22. Facchiano E, Scaringi S, Kianmanesh R, Sabate JM, Castel B, Flamant Y, Coffin B, Msika S. Laparoscopic hyperthermic intraperitoneal chemotherapy (HIPEC) for the treatment of malignant ascites secondary to unresectable peritoneal carcinomatosis from advanced gastric cancer. Eur J Surg Oncol. 2008; 34(2):154-8. Epub 2007 Jul 20.

23. Arbuck SG, Trave F, Douglass HO Jr, Nava H, Zakrzewski S, Rustum YM. Phase I and pharmacologic studies of intraperitoneal leucovorin and 5-fluorouracil in patients with advanced cancer. J Clin Oncol. 1986;4:1510-7.

24. Inaba Y, Arai Y, Matsueda K, Aramaki T, Kodera Y. Intractable massive ascites following radical gastrectomy, treatment with local intraperitoneal administration of OK-432 using a unified CT and fluoroscopy system. Australas Radiol. 2003;47:465-7.

25. Rosen HR, Jatzko G, Repse S, Potrc S, Neudorfer H, Sandbichler P, Zacherl J, Rabl H, Holzberger P, Lisborg P, Czeijka M. Adjuvant intraperitoneal chemotherapy with carbon-adsorbed mitomycin in patients with gastric cancer: results of a randomized multicenter trial of the Austrian Working Group for Surgical Oncology. J Clin Oncol. 1998;16:2733-8.

26. Nakayama N, Koizumi W, Tanabe S, Sasaki T, Saigenji K. A phase II study of combined chemotherapy with methotrexate, 5-fluorouracil, and low-dose cisplatin (MFP) for histologically diffuse-type advanced and recurrent gastric cancer (KDOG9501). Gastric Cancer. 2006;9:185-91.

27. Oshima T, Yamada R, Hatori S, Kunisaki C, Imada T. Pharmacokinetics of S-1 in patients with peritoneal dissemination of gastric cancer. Oncol Rep. 2006;16:361-6.

28. Ishido K, Koizumi W, Tanabe S, Higuchi K, Sasaki T, Katada C, Azuma M, Saigenji K, Futawatari N, Saegusa M. A patient with stage IV type 4 advanced gastric cancer who had a complete pathological response to short-term treatment with S-1 alone. Anticancer Drugs. 2008;19:921-5.

29. Sakamoto J, Matsui T, Kodera Y. Paclitaxel chemotherapy for the treatment of gastric cancer. Gastric Cancer. 2009;12:69-78.

30. Seewaldt VL, Greer BE, Cain JM, Figge DC, Tamimi HK, Brown WS, Miller SA. Paclitaxel (Taxol) treatment for refractory ovarian cancer: phase 2 clinical trial. Am J Obstet Gynecol. 1994;170:1666-71.

31. Du Bois A, Lück HJ, Buser K, Meerpohl HG, Sessa C, Klaassen U, Meden H, Bochtler H, Diergarten K. Extended phase 2 study 
of paclitaxel as a 3-h infusion in patients with ovarian cancer previously treated with platinum. Eur J Cancer. 1997;33:379-84.

32. Guastalla JP, Lhommé C, Dauplat J, Namer M, Bonneterre J, Oberling F, Pouillart P, Fumoleau P, Kerbrat P, Tubiana N. Taxol (paclitaxel) safety in patients with platinum pretreated ovarian carcinoma: an interim analysis of a phase 2 multicenter study. Ann Oncol. 1994;5:S33-8.

33. Vaughn DJ, Malkowicz SB, Zoltick B, Mick R, Ramchandani P, Holroyde C, Armstead B, Fox K, Wein A. Paclitaxel plus carboplatin in advanced carcinoma of the urothelium: an active and tolerable outpatient regimen. J Clin Oncol. 1998;16:255-60.

34. Hironaka S, Zenda S, Boku N, Fukutomi A, Yoshino T, Onozawa Y. Weekly paclitaxel as second-line chemotherapy for advanced or recurrent gastric cancer. Gastric Cancer. 2006;9:14-8.
35. Sebastian M, Kuemmel A, Schmidt M, Schmittel A. Catumaxomab: a bispecific trifunctional antibody. Drugs Today (Barc). 2009:45:589-97.

36. Lordick F, Ott K, Weitz J, Jäger D. The evolving role of catumaxomab in gastric cancer. Expert Opin Biol Ther. 2008; $8: 1407-15$.

37. Yamao T, Shimada Y, Shirao K, Ohtsu A, Ikeda N, Hyodo I, Saito H, Iwase H, Tsuji Y, Tamura T, Yamamoto S, Yoshida S. Phase II study of sequential methotrexate and 5-fluorouracil chemotherapy against peritoneally disseminated gastric cancer with malignant ascites: a report from the Gastrointestinal Oncology Study Group of the Japan Clinical Oncology Group, JCOG 9603 Trial. Jpn J Clin Oncol. 2004;34:316-22. 